7 Anonymous. Control of chronic pain. Br Med 7 1981;282:1095-6.

8 Snow $\mathrm{H}$. Opium and cocaine in the treatment of cancerous disease. $B$ Med f 1896;ii :718-9.

9 Twycross RG. Diamorphine and cocaine elixir BPC 1973. Pharmaceutical fournal $1974 ; 212: 153,159$.

10 Twycross RG, Fry DE, Wills PD. The alimentary absorption of diamorphine and morphine in man as indicated by urinary excretion studies. Br f Clin Pharmacol 1974;1:491-4.

${ }^{11}$ Kaiko RF, Wallenstein SL, Rogers AG, Grabinski PY, Houde RW. Analgesic and mood effects of heroin and morphine in cancer patients with postoperative pain. $N$ Engl f Med $1981 ; 304: 1501-5$.

12 Grabinski PY, Kaiko RF, Walsh TD, Foley KM, Houde RW. Morphine radioimmunoassay specificity before and after extraction of plasma and cerebrospinal fluid. $\mathcal{F}$ Pharm Sci 1983;72:27-30.

13 British Medical Association, The Pharmaceutical Society of Great Britain. British National Formulary, 5. London: British Medical Association, The Pharmaceutical Press, 1983.

${ }^{14}$ Beaumont IM. Stability study of aqueous solutions of diamorphine and morphine using HPLC. Pharmaceutical fournal $1982 ; 229: 39-41$.

\section{Primary aldosteronism: how hard should we look?}

Priorities may sometimes be decided quite innocently for the wrong reasons-and especially when the conceptually attractive competes with the unknown. Among the large section of the population at risk from high blood pressure only a small proportion will have a definable cause and the remainder are classified as having essential hypertension. Though both genetic and environmental influences are undoubtedly at work in these patients, no single cause can be found as yet-nor is it likely that any ever will be. The maxim "first seek a cause" is not absolute. A more relevant question is "how hard should I look ?" The answer to this question should be determined on the one hand by the benefits to the patient and on the other by the ease with which a cause can be detected.

With the most common detectable cause of hypertension the search is so simple that it should be carried out in every case: any woman of childbearing age should be asked whether she is taking the contraceptive pill. With renal and endocrine causes of hypertension, where the difficulty of making the diagnosis is greater, cost and benefit have to be weighed more carefully. We have to define the population we are investigating. Who is more likely to have a primary cause? What are the dangers of a misdiagnosis ? Are certain patients likely to benefit particularly if the diagnosis is made in them? In other words, though understanding the physiology and pathology of these conditions may help us to construct appropriate investigations and treatments, when planning what to do for an individual patient in an imperfect world with limited resources we have to start by analysing patient benefit. This is rather easier to do with renovascular hypertension ${ }^{12}$ than with primary aldosteronism, which is much rarer. Nevertheless some questions can be answered.

We do not know the overall incidence of primary aldosteronism. It is certainly less common than was originally postulated. ${ }^{3}$ The incidence is probably less than $1 \%,{ }^{45}$ though the more intensively primary aldosteronism is sought the more often it is encountered, and, like other forms of secondary hypertension, it is much more frequently encountered in specialist clinics than in unselected populations. The cardinal manifestations are a reduced serum potassium concentration, associated with raised plasma and urinary concentrations of aldosterone together with suppressed plasma renin activity. Suspicion is raised in most cases by a low serum potassium concentration, though this is more commonly a manifestation of secondary aldosteronism. Nevertheless, among 80 patients diagnosed at the Cleveland Clinic as having primary aldosteronism, no fewer than 22 had a normal serum potassium concentration at the time and 29 had plasma renin activities which were stimulated into the normal range by a low salt diet. ${ }^{6}$ The proportion of misdiagnoses falls with repeated measurement, though in one large series $11 \%$ of patients were persistently normokalaemic despite multiple measurements of serum potassium concentrations. ${ }^{5}$

By measuring either potassium or renin we seem likely, therefore, to miss a substantial number of patients with primary aldosteronism. Does it matter? The risks of primary aldosteronism are due to hypertension and potassium depletion. Since in most patients (though by no means all ${ }^{6}$ ) the raised blood pressure can be controlled with medication the most important clinical problem in primary aldosteronism is potassium depletion. Apart from causing unpleasant symptoms such as muscular weakness, hypokalaemia may be sufficiently severe to cause life threatening arrhythmias. Even more modest degrees of hypokalaemia may not be as innocent as was once thought. ${ }^{7}$ Hypokalaemia can usually be controlled by potassium retaining diuretics, ${ }^{8-10}$ but a relatively straightforward surgical procedure such as removal of an adenoma producing aldosterone has clear advantages provided it carries a high likelihood of success.

Unfortunately for our decision making process, the matter is not as simple as this analysis suggests. Firstly, primary aldosteronism is not a homogeneous condition. About $40 \%$ of patients have bilateral adrenal nodular hyperplasia rather than an adenoma producing aldosterone. ${ }^{6}$ These patients are best treated medically ${ }^{8}$; indeed, there are good physiological grounds for believing that nodular hyperplasia represents a form of essential hypertension in which the adrenal cortex is particularly sensitive to the action of angiotensin II in stimulating aldosterone secretion. ${ }^{112}$ The other clinically relevant fact about this group is that the biochemical changes tend to be less florid than in patients with adenomas, though there is considerable overlap between groups unless an elaborate statistical analysis is made of several variables ${ }^{5}$; even then it is difficult to apply such analysis to biochemical values obtained in different laboratories.

When, therefore, we investigate patients with normal or borderline serum potassium concentrations we may very well find that any primary aldosteronism we detect will be of a type which is best treated medically. If we insist on being reasonably sure that the diagnosis has not been missed we shall have to use intensive investigation requiring measurement of the aldosterone response to such procedures as dietary salt restriction and upright posture. ${ }^{6}$ The harder we look the more patients with aldosteronism we are likely to find, though the yield in terms of disorder correctable by surgery is likely to decline spectacularly the more widely we cast our net. If the dividing line between essential hypertension and primary aldosteronism due to nodular hyperplasia is as arbitrary as now appears likely we can no more expect to find an acid test for primary aldosteronism than for essential hypertension. In these circumstances screening for primary aldosteronism becomes pragmatic, and guidance should be based on likely outcome.

What guidance can be offered? Persistent severe hypokalaemia (serum potassium $<3.0 \mathrm{mmol}(\mathrm{mEq}) / \mathrm{l}$ ) occurring in either treated or untreated hypertensive patients requires investigation unless possible surgery is contraindicated on other grounds. Milder hypokalaemia (serum potassium $3 \cdot 0-3 \cdot 5 \mathrm{mmol} / \mathrm{l}$ ) in untreated patients is also probably worth investigating. Substantially fewer benefits are likely from 
investigating patients with inconsistent hypokalaemia or mild hypokalaemia taking thiazide diuretics, and in these circumstances the more reasonable approach is to attempt control with a regimen which includes a potassium retaining diuretic. Where either the raised blood pressure or the low serum potassium concentration cannot be adequately controlled more intensive investigation for primary aldosteronism in a special centre will be needed. With these guidelines the occasional patient with surgically correctable primary aldosteronism will be missed, but the cost of such an error in terms of patient morbidity or mortality will be extremely small.

Professor of Medicine,

J D SWALES

University of Leicester,

Leicester LE2 7LX

${ }^{1}$ McNeil BJ, Adelstein SJ. Measures of clinical efficacy: the value of case finding in hypertensive renovascular disease. $N$ Engl f Med 1975;293: 221-6.

${ }^{2}$ Swales JD. The hunt for renal hypertension. Lancet 1976;ii:930-2.

3 Conn JW, Cohen EL, Rovner DR, Nesbit RM. Normokalemic primary aldosteronism: a detectable cause of curable "essential hypertension." fAMA 1965;193:200-6.

- Berglund G, Andersson O, Wilhelmsen L. Prevalence of primary and secondary hypertension: studies in a random population sample. $\mathrm{Br} \mathrm{Med}$ F 1976;ii:554-6.

${ }^{6}$ Ferriss JB, Brown JJ, Fraser R, Lever AF, Robertson JI. Primary hyperaldosteronism. Clin Endocrinol Metab 1981;10:419-52.

- Bravo EL, Tarazi RC, Dustan HP, et al. The changing clinical spectrum of primary aldosteronism. Am $\mathcal{F}$ Med 1983;74:641-51.

7 Hollifield JW, Slaton PE. Thiazide diuretics, hypokalaemia and cardiac arrhythmias. Acta Med Scand 1981 ;suppl 647:67-73.

$\therefore$ Ferriss JB, Brown JJ, Fraser R, et al. Results of adrenal surgery in patients with hypertension, aldosterone excess, and low plasma renin concentrations. $B r$ Med 7 1975; i:135-8.

- Kremer D, Boddy K, Brown JJ, et al. Amiloride in the treatment of primary hyperaldosteronism and essential hypertension. Clin Endocrinol $1977 ; 7: 151-7$.

10 Ganguly A, Weinberger MH. Triamterene-thiazide combination: alternative therapy for primary aldosteronism. Clin Pharmacol Ther $1981 ; 30$ : 246-50.

1 Davies DL, Beevers DG, Brown JJ, et al. Aldosterone and its stimuli in normal and hypertensive man: are essential hypertension and primary hyperaldosteronism without tumour the same condition? 7 Endocrinol $1979 ; 81: 79 \mathrm{p}-91 \mathrm{p}$.

12 Wisgerhof M, Brown RD, Hogan MJ, Carpenter PC, Edis AJ. The plasma aldosterone response to angiotensin II infusion in aldosterone-producing adenoma and idiopathic hyperaldosteronism. f Clin Endocrinol Metab $1981 ; 52: 195-8$.

\section{Prostaglandins and menstrual disorders}

Prostaglandins have potent biological effects, but their instability and rapid metabolism make them short acting. They are produced but not stored by most living cells and act locally. Prostaglandins of the E and F groups cause myometrial contractions as well as having vasoactive properties. Prostacyclin is a potent vasodilator and inhibitor of platelet aggregation and may cause relaxation of the myometrium, ${ }^{1}$ while thromboxanes cause vasoconstriction and platelet aggregation. Non-steroidal anti-inflammatory drugs such as aspirin, indomethacin, naproxen, mefenamic acid, and ibuprofen exert their action by partially blocking an enzyme, cyclo-oxygenase, which converts arachidonic acid to the precursors of the various prostaglandins.

Menstruation is a hormonally controlled process of endometrial necrosis, shedding, and bleeding followed by haemostasis. Both the endometrium and myometrium have a considerable capacity to synthesise prostaglandins, particularly during the luteal phase of the cycle, ${ }^{2}$ when the presence of the cyclo-oxygenase enzyme in the endometrium may be shown histochemically. ${ }^{3}$ Prostaglandin $\mathrm{F}_{2^{\alpha}}$, a vasoconstrictor, is produced in large amounts by the uterus and it may trigger the ischaemic necrosis of menstruation. Vasodilatory prostaglandins such as prostaglandin $\mathrm{E}_{2}$ or prostacyclin are also produced by the uterus, and there is evidence that both are hormonally regulated. ${ }^{4}$ Increased menstrual bleeding might be due either to a reduction in the synthesis of prostaglandin $\mathrm{F}_{2^{\alpha}}$ or to an increase in the production of vasodilatory prostaglandins from endometrium or myometrium. Cultures of endometrium from women having normal menstrual cycles produce more prostaglandin $F_{2^{\alpha}}$ than prostaglandin $E_{2}$, whereas from women with menorrhagia endometrial culture produces more prostaglandin $\mathrm{E}_{2}$ than prostaglandin $\mathrm{F}_{2^{x}}$. Furthermore, endometrium from women with menorrhagia is more effective than the endometrium from women with normal menstrual loss in enhancing the production of prostacyclin by myometrium in vitro. ${ }^{6}$

Haemostasis in the uterus (as in the peripheral circulation) is dependent on platelet aggregation and deposition of fibrin. Menstrual blood has fewer platelets than peripheral blood, and their ability to aggregate and to synthesise prostaglandins in vitro is appreciably reduced. ${ }^{7}$ Menstrual blood is also lacking : in : fibrinogen and contains reduced amounts of antiplasmin. ${ }^{8}$

In the absence of a pathological disorder of the uterus menorrhagia is likely to be due to a combination of increased vasodilatation and impaired haemostasis. Treatment with a combined oestrogen-progestogen preparation or with danazol will reduce menstrual loss, but with the disadvantages of metabolic side effects and cost respectively. Alternatively, fibrinolytic activity may be reduced in the uterus by drugs, but these have some effect on the peripheral blood as well. In view of the possible consequences of such treatment attempts have been made to reduce menstrual loss by controlling the synthesis of prostaglandin with non-steroidal anti-inflammatory drugs. These drugs are non-specific in their inhibition of the production of prostaglandin since they block the synthesis of endoperoxides by the cyclo-oxygenase enzyme rather than the synthesis of specific prostaglandins. As long ago as 1976 Anderson $e t a l^{9}$ reported a reduction of menstrual loss when a drug inhibiting prostaglandin synthesis was taken during the days of menstrual bleeding. Reduced menstrual loss (assessed subjectively) has been reported with treatment with mefenamic acid. ${ }^{10}$ It is important to realise, however, that there seems to be no correlation between measured menstrual loss and the number of pads used. Subsequent double blind randomised placebo controlled crossover trials of mefenamic acid have shown a definite reduction in measured menstrual loss. The treatment has no effect in about one fifth of patients, and it seems impossible to predict who will respond and who will not. ${ }^{11} 12$

When treatment is given with non-steroidal antiinflammatory drugs they should be taken only immediately before and during the days of heavy menstrual bleeding. Side effects may include allergies, nausea, vomiting, dyspepsia, or, rarely, peptic ulceration. With such limited treatment, however, side effects are uncommon, and spasmodic dysmenorrhoea will also be relieved should this be present. In our present state of knowledge this form of medical treatment is worth trying, particularly in women with proved heavy bleeding who are anxious to avoid hysterectomy. Improved treatment will come only from more knowledge of the biochemical control of menstruation and the subsequent develop- 